\title{
Chapter 39 \\ Robust Optimization for the Discrete Time-Cost Tradeoff Problem with Cost Uncertainty
}

\author{
Öncü Hazır, Mohamed Haouari, and Erdal Erel
}

\begin{abstract}
Projects are subject to various sources of uncertainty that hamper reaching project targets; hence, it is crucial importance to use effective approaches to generate robust project schedules, which are less vulnerable to disruptions caused by uncontrollable factors. In this vein, this chapter examines analytical models and algorithms of robust multi-mode project scheduling, specifically, the robust discrete time-cost tradeoff problem (DTCTP). The models and algorithms presented in this chapter can support project managers from a wide range of industries in scheduling activities to minimize deviations from project goals. Furthermore, some surrogate measures that aim at providing an accurate estimate of the schedule robustness are developed and related experimental results are presented. Finally, some potential research areas are proposed and discussed.
\end{abstract}

Keywords Project management $・$ Robust optimization $\bullet$ Scheduling $\bullet$ Uncertain cost

\subsection{Introduction}

In order to better control and organize activities and reach organizational targets, today, organizations are becoming more and more project-driven. As a consequence, developing effective management techniques and applying them efficiently in projects attract the attention of both practitioners and academics. In this regard,

\footnotetext{
Ö. Hazır $(\bowtie)$

Faculty of Economics and Administrative Sciences, TED University, Ankara, Turkey e-mail: oncu.hazir@tedu.edu.tr

M. Haouari

Department of Mechanical and Industrial Engineering, College of Engineering, Qatar University, Doha, Qatar

e-mail: mohamed.haouari@qu.edu.qa

E. Erel

Faculty of Business Administration, Bilkent University, Ankara, Turkey

e-mail: erel@bilkent.edu.tr
} 
we focus on multi-mode project scheduling methods; specifically a well-known problem, the discrete time-cost tradeoff problem (DTCTP). A mode corresponds to a processing alternative of an activity, i.e., a different technology or a different resource assignment. These finite number of processing alternatives accommodate a compromise; faster ones being more costly. We examine two versions of the problem: deadline (DTCTP-D) and budget (DTCTP-B). In the deadline problem, total cost is minimized while terminating the project within the given deadline; whereas, the budget problem consists in minimizing the project duration without surpassing the given budget. Both of these multi-mode scheduling problems have practical application areas since they tackle the important time-cost tradeoff in processing activities.

DTCTP is a special case of multi-mode project scheduling. In single and multimode project scheduling literature, the majority of the studies assume knowledge of complete information. A detailed review of these studies can be found in Kolisch and Padman (2001), Herroelen (2005), Wȩglarz et al. (2011) and in Chap. 21 in the first volume of this handbook. Specializing on the problem, De et al. $(1995,1997)$ have respectively presented a survey on the problem and shown that the DTCTP is strongly NP-hard. For solution to optimality, Demeulemeester et al. (1996) provided a branch-and-bound algorithm; whereas Hazır et al. (2010a) developed a Benders decomposition based algorithm. On the other hand, to solve large instances, Akkan et al. (2005) and Vanhoucke and Debels (2007) proposed approximate approaches (see also Chap. 30 in the first volume of this handbook).

Although the majority of the studies in the field rely on the deterministic approaches, projects face various sources of uncertainty: some activities take more time than expected or some resources become unavailable due to reasons such as machine breakdowns. In order to reach project targets, hedging against uncertainty and proactive planning of the activities have become important. Stochastic programming, robust optimization, sensitivity analysis, parametric programming, and fuzzy programming are the main candidate optimization approaches to model and integrate project uncertainty in planning (Herroelen and Leus 2005).

In this chapter, we address robust scheduling of the DTCTP. In Sect. 39.2 we present and explain some robust optimization models, application areas. Later, in Sect. 39.3 we introduce relevant robustness measures. Finally, we discuss the rewarding topics and directions in Sect.39.4.

\subsection{Robust Optimization and Project Management}

Robust optimization aims to build solutions that are insensitive to data uncertainty. As the worst-case performance of the system is focused (Kouvelis and Yu 1997), some degree of pessimism is inherent in this approach. The most common approaches involve minmax cost and minmax regret objectives (Gabrel and Murat 2010). Respectively, maximum cost and maximum regret, which is the difference between the cost of the solution and optimal one across all scenarios, are minimized. They have been applied to well known combinatorial optimization problems 
(Aissi et al. 2009) and used in various application areas such as engineering and finance (Bertsimas et al. 2011).

In project management, following a scenario based approach, Yamashita et al. (2007) proposed robust optimization models for the resource availability cost problem, which consists in minimizing a cost function of resource availability. Each scenario corresponds to a realization of the uncertain durations. Differently, Cohen et al. (2007) used interval uncertainty in their recent robust scheduling study. Their study examines the effects of uncertainty on the continuous time-cost tradeoff problem. Similarly, Hazır et al. (2011) assumed interval uncertainty; but, focused on the discrete problem. For this problem, Klerides and Hadjiconstantinou (2010) addressed uncertainty in durations and used stochastic programming (see Chap. 36 of this book); whereas, Xu et al. (2012) applied fuzzy logic and presented a case study for an extension of the problem (see Chap. 59 of this book). Recently, Artigues et al. (2013) investigated minmax regret policies to model the robust version of the well-known resource-constrained project scheduling problem (see also Chap. 40 of this book).

When accurate records of past data are available to estimate probability distributions appropriately, stochastic programming has the advantage of incorporating this available information. However, as each project is unique, usually it is difficult to obtain reliable data. Therefore, in that sense, robust optimization is advantageous; furthermore, the project performance remains under control even in the worst-case conditions. In addition, the robust approach is distinctively different from sensitivity analysis, since it is proactive; it addresses uncertainty in the modeling phase.

Next, we summarize the modeling and solution approach of Hazır et al. (2011) for the robust DTCTP in the following section.

\subsubsection{Robust DTCTP}

Consider a project with a set of $n$ activities and a precedence graph in AoN (activityon-node) representation, $G=(V, E)$, where $V$, the node set, contains $n$ activities and two dummy nodes, 0 and $n+1$, that signify project start and termination. $E \subseteq$ $V \times V$, the arc set corresponds to precedence relationships among activities. Each activity $j \in V$ can be carried out in one of the $\left|\mathscr{M}_{j}\right|$ modes, where each one, $m \in \mathscr{M}_{j}$, is defined by duration $p_{j m}$ and a cost interval $\left[\underline{c}_{j m}, \bar{c}_{j m}\right]$. We define $c_{j m}$ and $\Delta c_{j m}$ as the most likely cost estimate and maximum cost increase from the nominal value; $\Delta c_{j m}=\bar{c}_{j m}-c_{j m}$. Using this notation, a mixed-integer programming formulation of the robust DTCTP-D can be given as follows:

$$
\begin{array}{ll}
\text { Min. } & \sum_{j=1}^{n} \sum_{m \in \mathscr{M}_{j}} c_{j m} x_{j m}+g(x) \\
\text { s.t. } & \sum_{m \in \mathscr{M}_{j}} x_{j m}=1 \quad(j=1, \ldots, n)
\end{array}
$$




$$
\begin{aligned}
& C_{j}-C_{i}-\sum_{m \in \mathscr{M}_{j}} p_{j m} x_{j m} \geq 0 \quad((i, j) \in E) \\
& C_{n+1} \leq \bar{d} \\
& g(x)=\operatorname{Max} .\left\{\sum_{j \in V} \sum_{m \in \mathscr{M}_{j}} \Delta c_{j m} x_{j m} u_{j}: \sum_{j \in V} u_{j} \leq \Gamma, u_{j} \in\{0,1\}\right\}
\end{aligned}
$$

In the formulation, the continuous decision variable, $C_{j}$, represents the completion time of activity $j$, whereas the binary variable $x_{j m}$ assigns mode $m \in \mathscr{M}_{j}$ for activity $j$, i.e., $x_{j m}=1$. With the objective of minimizing the total cost (Eq. 39.1), a unique mode should be chosen for each activity (Eq.39.2), while precedence constraints (Eq. 39.3) must be satisfied and the deadline, notated with $\bar{d}$, must not be exceeded (Eq. 39.4).

Note that maximum cost deviation is formulated with function $g(x)$ (Eq. 39.5). In this function, binary vector $u$ is used to determine the activities (at most $\Gamma$ ) that will be processed with the worst-case cost values, i.e., $\left\{j: u_{j}=1\right\}$. Note that if $\Gamma=0$, the deterministic DTCTP-D is obtained, whereas high values of this parameter represent risk-averse decision making behavior.

Based on the formulation given above, Hazır et al. (2011) examined three robust versions of DTCTP-D. Model formulations, exact and approximate solution approaches, and computational results were presented.

The first version assumes that uncertainty could be defined as a cardinality constrained set (Bertsimas and Sim 2003; Bertsimas et al. 2011); that is, only a subset of coefficients ( $\Gamma$ of them) reach the worst case values. For solution, Benders decomposition, which has been widely used in various combinatorial optimization problems including project and airline scheduling, was used (Erenguc et al. 1993; Li and Womer 2009; Sherali et al. 2010; Chap. 27 in the first volume of this handbook).

Regarding robustness of project schedules, an important factor to be investigated is total slack, which is the amount of time by which the activity completion could be delayed without delaying the project termination (see Garaix et al. 2013 and Chap. 41 of this book for calculating the slack values in case of interval uncertainty for activity durations). Note that larger slacks provide flexibility in scheduling and resource allocation. Moreover, activity costs and durations are interdependent, as they both depend on the amount of resource allocation. During project execution, non-critical activities could be started later or less amount of resources could be allocated to these activities. Therefore, in achieving the cost targets, these activities constitute less risk when compared to the critical ones. Hence, assigning the worstcase costs to activities with ample slacks could be unrealistically pessimistic. On the other hand, in case of disruptions, for finishing critical activities on time, more monetary resources might have to be allocated.

In this regard, in the second version, Hazir et al. (2011) considered only the potentially critical activities to define the possible cost deviations (criticalitybased robust model). These activities are identified based on the total slack/activity 
duration ratio. The smaller the ratio, the more critical the activity. Lastly, a third model that gives priority to critical activities over non-critical ones in calculating cost deviations was also developed. As could be expected, the second and third models are more difficult to solve; hence high quality approximate solutions were sought using tabu search.

When these approaches are compared, the first approach is the most pessimistic one and performs well under extreme scenarios. However, the second one allocates larger project buffers at the end, which has been shown to be efficient in case of variations in activity durations. The third one results in total slacks distributed more evenly among activities.

\subsubsection{An Application Area}

As an application area, Hazır et al. (2011) cite Build-Operate-Transfer (BOT) projects, in which a public service or an infrastructure investment is undertaken and operated for a specific period of time by a private firm and then transferred to a public institution. The BOT contracts offer several mutual advantages. For the public sector, they offer an alternative financing mechanism to carry out large investment projects and encourage foreign investment inflow. On the other hand, as the client is usually the government, they reduce demand and credit risks of the private sector. To have more information about the characteristics of these contracts, we refer to the paper of McCarthy and Tiong (1991).

One of the application areas of BOT model are airport and construction projects. The private enterprise constructs the airport or harbor and operates it for a predefined period of time and then transfers the right to operate to the public. The enterprise can extend the operating period via completing the construction earlier. Early completion could be highly profitable. For that reason, in case of deviations from the baseline plan, extra resources are commonly used to speed up the activities. However, these additional allocations create cost uncertainty. Protection against deviations in total cost and project duration then becomes the key concern of project managers and in this regard, decision support systems that involve robust planning approaches are valuable. In addition, considering multiple alternatives for processing activities fit the characteristics of these types of contracts.

\subsection{Robustness Measures}

In addition to algorithm design, another important issue in robust scheduling is to evaluate the robustness of schedules and the comparison of scheduling algorithms based on the robustness of the designs produced (example studies are: Goren and Sabuncuoglu 2008 for machine scheduling, and Chtourou and Haouari 2008 for resource-constrained project scheduling problem). For this well-known 


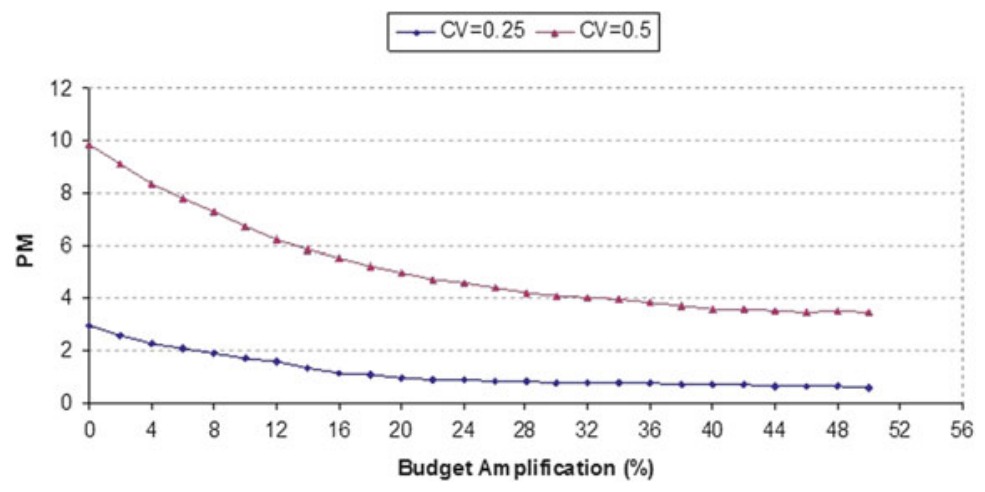

Fig. 39.1 The relationship between budget amplification and performance measure

project management problem, Al-Fawzan and Haouari (2005) followed a biobjective approach by optimizing both robustness and project makespan. Regarding DTCTP, Hazır et al. (2010b) proposed nine time-based measures, which could be categorized as:

1. Average Slack

2. Weighted Slack

3. Slack Utility Function

4. Dispersion of Slacks

5. Percentage of Potentially Critical Activities

6. Project Buffer Size

In order to evaluate these measures, a simulation study was carried out for a large number project networks. As a result of the simulation, project buffer size and weighted slack (weights referring to the number of immediate successors of the activity) were found to be best estimates of schedule robustness.

Based on these results, a two phase scheduling algorithm to place a project buffer with minimal cost was proposed (inserting project buffers instead of augmenting the activity durations is one of the important characteristics of critical chain project scheduling). To accomplish that, an inflated budget is used; the initial budget is augmented so that the scheduling algorithm produces a schedule with a shorter termination time. Then, the effect of this budget amplification is experimentally tested using a subset of instances of Akkan et al. (2005). One of the performance measures (PM) is the average delay in the project completion time as percentage of the project deadline. That is, using this measure, schedules that exhibit less delays in case of disruptions are considered to be more robust.

In the simulation based analysis, first, a budget amplification rate is set and a baseline schedule with a project buffer is generated using this new budget value. Then, for each instance of the test bed, perturbations in processing times were randomly generated and the resulting project termination time is noted. Figure 39.1 shows some of the results. The coefficient of variation (CV) values 0.25 and 0.5 
characterize small and moderate variability in activity durations. In case of low variability, PM could be significantly improved with small budget increases. For instance, with a $6 \%$ budget increase $(\eta=0.06)$, the average delay decreased from about 3 to $2 \%$. Further increasing the budget accompanies a decrease in the marginal gain. However, in case of higher variability, improvement in project delays are more drastic, but larger budget increases are needed to have a stabilized pattern and eliminate tardiness.

\subsection{Discussion on Future Research Directions}

Possible future research topics are summarized as follows:

1. Project management has mainly focused on the variations in activity durations. However, variations in resource requirements and resource availability could also have significant consequences. Studies on variability of resource are scarce; however, considering real instances there is a need for further analysis and analytical models. In this regard, robust versions of multi-mode resourceconstrained scheduling models should be investigated.

2. For multi-mode scheduling, minmax and minmax regret models could also be studied. These models are usually difficult to solve, hence the development of efficient solution algorithms, especially approximate ones for large instances, is important and interesting for further research.

3. Other than well-known minmax algorithms, some recent approaches could be used to model robust project scheduling problems. Especially two of them are appealing:

- The $b w$ robustness proposed by Gabrel et al. (2013) aims to obtain objective values smaller than $w$ in all scenarios and below the target value of $b$ as much as possible $b<w$ (in a minimization problem).

- Lexicographic $\alpha$-robustness reduces the influence of worst-case scenario and incorporates some tolerance with the help of the threshold $\alpha$ (Kalai et al. 2012). Project scheduling problems would be a relevant application area for these approaches; but establishing efficient solution algorithms for large scale projects stays to be arduous and important.

4. The majority of project scheduling studies optimizes a single performance measure, such as minimization of the project completion time. However, schedules that are developed with traditional algorithms may result in poor performances in case of disruptions. Therefore, multi criteria optimization models that combine a time or cost based objective with a robustness criterion are promising research topics and are better adapted to the requirements of industry.

5. Modeling robust versions of multi project environments systems may constitute a rewarding research field. 
6. If robustness with respect to project completion time is taken as the fundamental optimization criterion, there will be tendency to schedule activities at their earliest start times (see experimental study and results of Tian and Demeulemeester 2013 on road runner scheduling). On the other hand, late starts have the advantage of decreasing WIP inventory costs, which is one of the key elements of just-in-time (JIT) management philosophy. Moreover, in large capital-intensive projects, cash flow management is vital and delaying cash outlays as much as possible is usually preferred. Therefore, an optimal compromise between project costs and robustness has to be achieved. Modeling this relationship between robust scheduling and project costs is an interesting research topic.

7. Combining reactive and robust project scheduling improves project performance. This combined approach is new in scheduling literature and referred as "proactive-reactive scheduling". This approach protects against disruptions through the combination of a proactive scheduling and a reactive improvement procedure. The baseline schedule could be created by the maximization of a robustness measure so that it involves enough safety time to absorb anticipated disruptions. Even though this baseline schedule will be insensitive to some extent, all possible disruptions might not be anticipated. For this reason, it is better to include reactive scheduling as the second protection mechanism to prevent large performance deviations due to disruptions.

8. Specific project contract types should be further investigated and specific robust planning models approaches for these projects could be developed. One example is the BOT project types, which we have already mentioned. Recently, these types of contracts have been widely signed especially in developing countries.

9. Finally, developing robust project scheduling as a model base of a DSS to assist managers is crucial. Embedding these DSS tools in a commercial software package such as in the widely used Microsoft Project system would be of considerable help to project managers. At this point, combining these scheduling algorithms with efficient project control modules that define intervening strategies in case of disruptions will be a promising application area. We refer to the study of Hazir and Schmidt (2013) as an example to integrate scheduling and control functions in multi-mode projects.

\subsection{Conclusions}

Robustness of solutions has been increasingly attracting the attention of researchers and practitioners in operations research and related fields. In this chapter, we examine and discuss application of robust optimization on multi-mode project scheduling. We present mathematical models to generate schedules and measures to assess their robustness. Models and extensions have practical meaning in the sense that they could constitute the model basis of a decision support system on this subject. Furthermore, discussion on research directions can facilitate identifying research topics that have theoretical values or practical applications. 


\section{References}

Aissi H, Bazgan C, Vanderpooten D (2009) Min-max and min-max regret versions of combinatorial optimization problems: a survey. Eur J Oper Res 197(2):427-438

Akkan C, Drexl A, Kimms A (2005) Network decomposition-based benchmark results for the discrete time-cost tradeoff problem. Eur J Oper Res 165(2):339-358

Al-Fawzan M, Haouari M (2005) A bi-objective model for robust resource-constrained project scheduling. Int J Prod Econ 96(2):175-187

Artigues C, Leus R, Talla Nobibon F (2013) Robust optimization for resource-constrained project scheduling with uncertain activity durations. Flex Serv Manuf J 25:175-205

Bertsimas D, Sim M (2003) Robust discrete optimization and network flows. Math Program 98:49_ 71

Bertsimas D, Brown D, Caramanis C (2011) Theory and applications of robust optimization. SIAM Rev 53(3):464-501

Chtourou H, Haouari M (2008) A two-stage-priority-rule-based algorithm for robust resourceconstrained project scheduling. Comput Ind Eng 55(1):183-194

Cohen I, Golany B, Shtub A (2007) The stochastic time-cost tradeoff problem: a robust optimization approach. Networks 49(2):175-188

De P, Dunne EJ, Ghosh JB, Wells CE (1995) The discrete time-cost tradeoff problem revisited. Eur J Oper Res 81(2):225-238

De P, Dunne EJ, Ghosh JB, Wells CE (1997) Complexity of the discrete time/cost trade-off problem for project networks. Oper Res 45:302-306

Demeulemeester EL, Herroelen WS, Elmaghraby SE (1996) Optimal procedures for the discrete time/cost trade-off problem in project networks. Eur J Oper Res 88(1):50-68

Erenguc SS, Tufekci S, Zappe CJ (1993) Solving time/cost trade-off problems with discounted cash flows using generalized benders decomposition. Nav Res Log 40(1):25-50

Gabrel V, Murat C (2010) Robustness and duality in linear programming. J Oper Res Soc 61:12881296

Gabrel V, Murat C, Wu L (2013) New models for the robust shortest path problem: complexity, resolution and generalization. Ann Oper Res 207(1):97-120

Garaix T, Artigues C, Briand C (2013) Fast minimum float computation in activity networks under interval uncertainty. J Sched 16(1):93-103

Goren S, Sabuncuoglu I (2008) Robustness and stability measures for scheduling: single-machine environment. IIE Trans 40(1):66-83

Hazır O, Schmidt K (2013) An integrated scheduling and control model for multi-mode projects. Flex Serv Manuf J 25(1-2):230-254

Hazır O, Haouari M, Erel E (2010a) Discrete time/cost trade-off problem: a decomposition-based solution algorithm for the budget version. Comput Oper Res 37(4):649-655

Hazır O, Haouari M, Erel E (2010b) Robust scheduling and robustness measures for the discrete time/cost trade-off problem. Eur J Oper Res 207(2):633-643

Hazır O, Erel E, Gunalay Y (2011) Robust optimization models for the discrete time/cost trade-off problem. Int J Prod Econ (1):87-95

Herroelen W (2005) Project scheduling: theory and practice. Prod Oper Manag 14(4):413-432

Herroelen W, Leus R (2005) Project scheduling under uncertainty: survey and research potentials. Eur J Oper Res 165(2):289-306

Kalai R, Lamboray C, Vanderpooten D (2012) Lexicographic $\alpha$ robustness: an alternative to minmax criteria. Eur J Oper Res 220(3):722-728

Klerides E, Hadjiconstantinou E (2010) A decomposition-based stochastic programming approach for the project scheduling problem under time/cost trade-off settings and uncertain durations. Comput Oper Res 37(12):2131-2140

Kolisch R, Padman R (2001) An integrated survey of deterministic project scheduling. Omega-Int J Manag S 29(3):249-272 
Kouvelis P, Yu G (1997) Robust discrete optimization and its applications. Nonconvex optimization and its applications, vol 14. Kluwer Academic, Dordrecht

Li H, Womer K (2009) Scheduling projects with multi-skilled personnel by a hybrid MILP/CP benders decomposition algorithm. J Sched 12:281-298

McCarthy SC, Tiong RLK (1991) Financial and contractual aspects of build-operate-transfer projects. Int J Proj Manag 9(4):222-227

Sherali HD, Bae KH, Haouari M (2010) Integrated airline schedule design and fleet assignment: polyhedral analysis and benders decomposition approach. INFORMS J Comput 22(4):500-513

Tian W, Demeulemeester E (2013) On the interaction between roadrunner or railway scheduling and priority lists or resource flow networks. Flex Serv Manuf J 25:145-174

Vanhoucke M, Debels D (2007) The discrete time/cost trade-off problem under various assumptions exact and heuristic procedures. J Sched 10:311-326

Wȩglarz J, Józefowska J, Mika M, Waligóra G (2011) Project scheduling with finite or infinite number of activity processing modes: a survey. Eur J Oper Res 208(3):177-205

Xu J, Zheng H, Zeng Z, Wu S, Shen M (2012) Discrete time-cost-environment trade-off problem for large-scale construction systems with multiple modes under fuzzy uncertainty and its application to Jinping II hydroelectric project. Int J Proj Manag 30(8):950-966

Yamashita D, Armentano VA, Laguna M (2007) Robust optimization models for project scheduling with resource availability cost. J Sched 10:67-76 